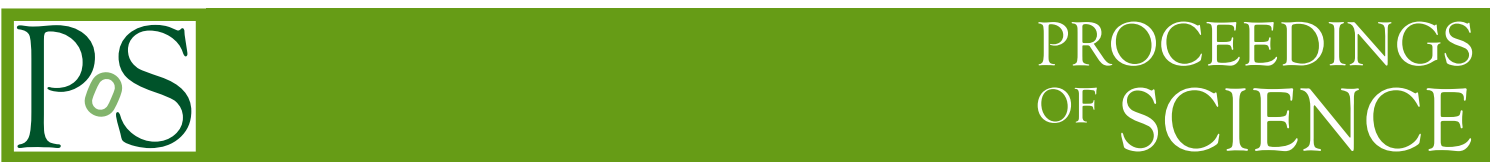

Massive neutrinos in a grounds-up approach

\title{
Shaouly Bar-Shalom*
}

Physics Department, Technion-Institute of Technology, Haifa 32000, Israel

E-mail: shaoulyephysics.technion.ac.il

\section{David Atwood}

Dept. of Physics and Astronomy, Iowa State University, Ames, IA 50011, USA

E-mail: atwoodeiastate.edu

\section{Amarjit Soni}

High Energy Theory Group, Brookhaven National Laboratory, Upton, NY 11973, USA

E-mail: soni@bnl.gov

\begin{abstract}
We examine neutrino oscillations in a two Higgs doublet model (2HDM) in which the second doublet couples only to the third generation right-handed up-fermions, i.e., to $t_{R}$ and $N_{3}$ which is the heaviest right-handed Majorana neutrino. The inherently large $\tan \beta$ of this model can naturally account for the large top-quark mass and, based on a quark-lepton similarity ansatz, when embedded into a seesaw mechanism it can also account for the observed neutrino masses and mixing angles giving a very small $\theta_{13}:-0.96^{0} \lesssim \theta_{13} \lesssim 1.36^{0}$ at $99 \% \mathrm{CL}$, and a very restrictive prediction for the atmospheric mixing angle: $42.9^{0} \lesssim \theta_{\text {atm }} \lesssim 45.2^{0}$ at $99 \% \mathrm{CL}$. The large value of $\tan \beta$ also sets the mass scale of the heaviest right-handed Majorana neutrino $N_{3}$ and triggers successful leptogenesis.
\end{abstract}

International Europhysics Conference on High Energy Physics

July 21st - 27th 2005

Lisboa, Portugal

${ }^{*}$ Speaker. 


\section{Introduction}

In the past decade we have witnessed two remarkable findings: (i) the discovery of the topquark which turned out to be enormously heavy compared to all the other fermions: $m_{t} \sim 175 \mathrm{GeV}$, i.e., "weighing" almost as much as a Gold atom!, and (ii) the discovery of neutrino oscillations implying that neutrinos are massive with a typical mass in the sub-eV range, i.e., $m_{v}$ is more than 12 orders of magnitudes smaller than $m_{t}$. This two monumental discoveries of the 90's present us with the pressing challenge of reconciling the apparent enormous hierarchy in the masses of fundamental fermions.

A possible resolution to this huge hierarchy between $m_{v}$ and $m_{t}$ may be encoded within the following triple-relation between $m_{v}, m_{t}$ (or the Electroweak scale) and the GUT mass-scale $M_{G U T} \sim 10^{16} \mathrm{GeV}:$

$$
m_{v} \sim \frac{m_{t}^{2}}{M_{G U T}} .
$$

Indeed, the beautiful seesaw mechanism dictates that (see also next sections):

$$
m_{v} \sim \frac{m_{D}^{2}}{M_{v_{R}}}
$$

where $m_{D}$ is a Dirac neutrino mass term and $M_{v_{R}}$ is the mass of heavy right-handed Majorana neutrinos. Thus, based on the seesaw formula in Eq. 1.2, the triple-relation in Eq. 1.1 stands as a very strong hint for Dirac neutrino masses of $m_{D} \sim \mathscr{O}\left(m_{t}\right)$ and for the existence of super-heavy right-handed Majorana neutrinos with a typical mass of $M_{v_{R}} \sim \mathscr{O}\left(M_{G U T}\right)$.

In this work [1] we seriously take the triple-relation in Eq. 1.1 at "face-value", suggesting that the impressive findings in the neutrino sector are closely related to the heaviness of the topquark. In particular, we construct a model that, based on a grounds-up approach, explicitly yields the triple-relation between the large $m_{t}$, the observed $v$-oscillation data (i.e., masses and mixing angles) and the super-heavy mass scale of the right-handed Majorana neutrinos. In addition, our model can rigger successful leptogenesis which can account for the observed Baryon asymmetry in the universe.

Our model is a two Higgs doublet model (2HDM) which treats the 3rd generation neutrino in a completely analogous manner to the top-quark. We have, therefore, named our model "the 2HDM for the 3rd generation" (3g2HDM).

\section{The two Higgs doublet model for the 3rd generation (3g2HDM)}

The 3g2HDM extends the idea of the so called "2HDM for the top-quark" (t2HDM) [2] to the leptonic sector. In particular, as in the t2HDM, we assume that $\phi_{t}$ [the Higgs doublet with a much larger vacuum expectation value (VEV)] couples only to the top-quark and to the 3rd generation right-handed Majorana neutrino, while the other Higgs doublet $\phi_{f}$ (with a much smaller VEV) couples to all the other fermions. The large mass hierarchy between the top-quark and all other fermions is then viewed as a consequence of $v_{t} / v_{f} \equiv \tan \beta>>O(1)$, which, therefore, becomes the "working assumption" of our 3g2HDM. 
The Yukawa interaction Lagrangian of our 3g2HDM takes the form:

$$
\mathscr{L}_{Y}=-Y^{d} \bar{Q}_{L} \phi_{f} d_{R}-Y_{1}^{u} \bar{Q}_{L} \tilde{\phi}_{f} u_{R}-Y_{2}^{u} \bar{Q}_{L} \tilde{\phi}_{t} u_{R}-Y^{e} \bar{L}_{L} \phi_{f} \ell_{R}-Y_{1}^{v} \bar{L}_{L} \tilde{\phi}_{f} N-Y_{2}^{v} \bar{L}_{L} \tilde{\phi}_{t} N+\text { h.c. },
$$

where $N$ are right-handed Majorana neutrinos with a mass $M_{N}^{i j} N_{i} N_{j} / 2, Q$ and $L$ are the usual quark and lepton doublets and the following Yukawa textures are assumed [1]

$$
Y_{1}^{u, v} \equiv\left(\begin{array}{ccc}
a^{u, v} & b^{u, v} & 0 \\
a^{u, v} & b^{u, v} & 0 \\
0 & \delta b^{u, v} & 0
\end{array}\right), Y_{2}^{u, v} \equiv\left(\begin{array}{ccc}
0 & 0 & 0 \\
0 & 0 & c^{u, v} \\
0 & 0 & c^{u, v}
\end{array}\right),
$$

such that, in both the quark and leptonic sectors, $\phi_{t}$ couples only to the third generation righthanded up-fermions. Note also that $m_{D}, m_{u}=v_{f}\left(Y_{1}^{v, u}+\tan \beta Y_{2}^{v, u}\right) / \sqrt{2}$, where $m_{D}, m_{u}$ are the Dirac mass matrices of the neutrinos and up-quarks, respectively.

\section{Neutrino oscillations in the 3g2HDM}

In the basis where $M_{N}$ is diagonal, $M_{N}=M \cdot \operatorname{diag}\left(\varepsilon_{M 1}, \varepsilon_{M 2}, \varepsilon_{M 3}\right)$, we obtain from the seesaw mechanism formula $m_{v}=-m_{D} M_{N}^{-1} m_{D}^{T}$ :

$$
m_{v}=m_{v}^{0}\left(\begin{array}{ccc}
\varepsilon & \varepsilon & \delta \bar{\varepsilon} \\
\cdot & \varepsilon+\omega & \delta \bar{\varepsilon}+\omega \\
\cdot & \cdot & \delta^{2} \bar{\varepsilon}+\omega
\end{array}\right)
$$

where

$$
m_{v}^{0} \equiv \frac{\left(v_{1}\right)^{2}}{2 M}, \varepsilon \equiv \frac{a^{2}}{\varepsilon_{M 1}}+\frac{b^{2}}{\varepsilon_{M 2}}, \bar{\varepsilon} \equiv \varepsilon-\frac{a^{2}}{\varepsilon_{M 1}}, \omega \equiv \frac{c^{2} t_{\beta}^{2}}{\varepsilon_{M 3}} .
$$

In the following we will adopt a quark-lepton similarity Ansatz (perhaps motivated by GUT scenarios): $a^{u} \sim a^{v} \equiv a, b^{u} \sim b^{v} \equiv b$ and $c^{u} \sim c^{v} \equiv c$, with $a \sim O\left(10^{-3}\right), b \sim O\left(10^{-1}\right), c \sim O(1)$ which, in our model, follows from the up-quark sector since $a^{u} v_{f} \sim O\left(m_{u}\right), b^{u} v_{f} \sim O\left(m_{c}\right)$ and $m_{t} \sim O\left(c^{u} v_{f} \tan \beta\right)$. Then, diagonalizing the light-neutrinos mass matrix in Eq. 3.1, we find that in the normal mass-hierarchy scheme, i.e., $m_{1}<<m_{2}<<m_{3}$, [1]:

- The mass of the heaviest light-neutrino follows the triple relation in Eq. 1.1:

$$
m_{3} \sim \frac{m_{t}^{2}}{M_{N_{3}}},
$$

where $M_{N_{3}} \sim M_{G U T}$ is the mass of the 3 rd and heaviest right-handed Majorana neutrino.

- Performing a minimum $\chi^{2}$ analysis with respect to each of the oscillation parameters $\theta_{13}, \theta_{\text {atm }} \equiv$ $\theta_{23}, \theta_{\text {sol }} \equiv \theta_{12}$ and $\Delta m_{\text {atm }}^{2}, \Delta m_{\text {sol }}^{2}$, our 3g2HDM yields the following $99 \% \mathrm{CL}$ allowed ranges for the mixing parameters:

$$
\begin{aligned}
& 28.0^{0} \lesssim \theta_{\text {sol }} \lesssim 36.0^{0} \quad 99 \% \mathrm{CL}, \\
& 1.0 \cdot 10^{-3}(\mathrm{eV})^{2} \lesssim \Delta m_{\text {atm }}^{2} \lesssim 3.7 \cdot 10^{-3}(\mathrm{eV})^{2} \quad 99 \% \mathrm{CL}, \\
& 7.3 \cdot 10^{-5}(\mathrm{eV})^{2} \lesssim \Delta m_{\text {sol }}^{2} \lesssim 9.1 \cdot 10^{-5}(\mathrm{eV})^{2} \quad 99 \% \mathrm{CL},
\end{aligned}
$$


with a very restrictive prediction for $\theta_{13}$ and the atmospheric mixing angle:

$$
\begin{aligned}
-0.96^{0} & \lesssim \theta_{13} \lesssim 1.36^{0} \quad 99 \% \mathrm{CL}, \\
42.9^{0} \lesssim \theta_{\text {atm }} & \lesssim 45.2^{0} \quad 99 \% \mathrm{CL} .
\end{aligned}
$$

- The mass-spectrum of the heavy Majorana neutrinos (subject to the constraints coming from oscillation data) becomes:

$$
M_{N_{3}} \sim 100 M, M_{N_{2}} \sim 0.01 M, M_{N_{1}}>>10^{-6} M,
$$

with $M \sim 10^{13} \mathrm{GeV}$.

\section{Leptogenesis in the $3 \mathrm{~g} 2 \mathrm{HDM}$}

A CP-asymmetry, $\varepsilon_{N_{i}}$, in the decay $N_{i} \rightarrow \ell \phi_{j}$ can generate the lepton asymmetry [3]:

$$
n_{L} / s=\varepsilon_{N_{i}} Y_{N_{i}}\left(T>>M_{N_{i}}\right) \eta
$$

where $Y_{N_{i}}\left(T>>M_{N_{i}}\right)=135 \zeta(3) /\left(4 \pi^{4} g_{*}\right)$ and $g_{*}$ being the effective number of spin-degrees of freedom in thermal equilibrium. Also, $\eta$ is the "washout" parameter (efficiency factor) that measures the amount of deviation from the out-of-equilibrium condition at the time of the $N_{i}$ decay. This lepton asymmetry can then be converted into a baryon asymmetry through nonperturbative spheleron processes. In our case (i.e., two scalar doublets) we obtain:

$$
n_{B} / s \sim-1.4 \times 10^{-3} \varepsilon_{N_{i}} \eta
$$

As seen from Eq. 3.6, our 3g2HDM can lead to a hierarchical mass spectrum for the heavy Majorana neutrinos, $M_{N_{1}}<<M_{N_{2}}<<M_{N_{3}}$. In this case, only the CP-asymmetry produced by the decay of $N_{1}$ survives, i.e., $\varepsilon_{N_{i}} \rightarrow \varepsilon_{N_{1}}$. Calculating the CP-asymmetry $\varepsilon_{N_{1}}$ and the corresponding washout factor $\eta$ we obtain [1]:

$$
\frac{n_{B}}{s} \sim 10^{-17} \tan ^{2} \beta \frac{\sqrt{\Delta m_{\text {sol }}^{2}}}{2 m_{t}^{2}} \varepsilon M_{N_{1}}\left(\frac{M_{N_{1}}}{\mathrm{GeV}}\right)^{1.2} \sin 2\left(\theta_{b}-\theta_{a}\right) .
$$

where the CP-phases arise from the possible complex entries in $Y_{1}^{v}: a=|a| e^{i \theta_{a}}$ and $b=|b| e^{i \theta_{b}}$. Eq. 4.3 has to be compared with the observed baryon to photon number ratio $n_{B} / n_{\gamma} \sim 6 \times 10^{-10}$, implying $n_{B} / s \sim 8.5 \times 10^{-11}$. For example, taking $\varepsilon \sim 0.5$ and $\Delta m_{\text {sol }}^{2} \sim 8.2 \cdot 10^{-5} \mathrm{eV}^{2}$ (these values are consistent with the observed oscillation data), along with $\tan \beta \sim 10$ and $m_{t} \sim 170 \mathrm{GeV}$, Eq. 4.3 reproduces the observed baryon asymmetry for e.g., $M_{N_{1}} \sim 10^{10} \mathrm{GeV}$ and $\sin 2\left(\theta_{b}-\theta_{a}\right) \sim 0.1$, or for $M_{N_{1}} \sim 3.6 \cdot 10^{9} \mathrm{GeV}$ if $\mathrm{CP}$ is maximally violated in the sense that $\sin 2\left(\theta_{b}-\theta_{a}\right) \sim 1$.

\section{References}

[1] For more details see, D. Atwood, S. Bar-Shalom and A. Soni, [hep-ph/ 0502234 ].

[2] A.K. Das and C. Kao, Phys. Lett. B372, (1996) 106.

[3] See e.g., P. Di Bari, [hep-ph / 0406115$]$; T. Hambye, [hep-ph / 0412053$].$ 